\title{
Approach to the nonequilibrium time-periodic state in a steady shear flow model
}

\author{
Janka Petravic and Denis J. Evans \\ Research School of Chemistry, Australian National University, \\ Canberra, Australian Capital Territory 0200, Australia
}

\begin{abstract}
The standard nonequilibrium molecular dynamics algorithm for steady shear flow (SLLOD ) employs Lees-Edwards periodic boundary conditions. It is not widely known that these boundary conditions make the system non-autonomous. The "steady state" shear stress is in fact time periodic. The standard response theory derivations for steady shear do not take proper account of these non-autonomous terms. In this paper we correct this deficiency. We show that these non-autonomous terms invalidate the GreenKubo relation for the finite frequency shear viscosity of fluids under Lees-Edwards periodic boundary conditions.
\end{abstract}

PACS: $05.20 .-\mathrm{y}, 47.11 .+\mathrm{j}$ 


\section{INTRODUCTION}

The standard algorithm for calculating the shear viscosity and other viscometric properties of fluids involves simulating planar Couette-Taylor flow with Lees-Edwards periodic boundary conditions [1]. In the absence of thermostatting this technique is known to be exact arbitrarily far from equilibrium since the equations of motion, the socalled SLLOD equations, are equivalent to applying Newton's equations to a system which initially is characterised by the local equilibrium distribution for shear flow [1]. The use of Lees-Edwards periodic boundary conditions is simply a generalisation of the usual time independent periodic boundary conditions so that they apply to the continuous shearing motion of the periodic 'lattice' of unit cells. There is simply no other way of incorporating shear in a homogeneous periodic system.

A non-equilibrium steady state can be achieved only if a thermostat, such as Gauss' isokinetic constraint, is added to the equations of motion. The heat generated by the shear is then removed (or added) and the peculiar kinetic energy remains constant at all times. In the absence of any actual boundaries it is clear that heat removal must be accomplished in a non-physical manner. However, in the linear regime close to equilibrium, theorems are known about the properties of Gaussian thermostatted systems. We know that in this regime the viscosity and indeed the fluid structure, is independent of the thermostatting mechanism [1]. Even in the nonlinear regime it is known that many thermostatting mechanisms all generate the same fluid structure and properties (at least to first order in $\mathrm{N}$, the number of particles).

Comparisons have been performed of fluid properties in the nonlinear regime for homogeneously thermostatted systems and for more realistic wall-thermostatted systems. The results have shown that even when the shear is sufficiently large that $30 \%$ shear thinning has ocurred, there is no detectable difference between the local properties of the inhomogeneous system and the global properties of the homogeneous, Gaussian thermostatted, SLLOD system [2]. 
The thermostatted SLLOD algorithm with Lees-Edwards periodic boundary conditions and a reversible deterministic thermostat, is now the standard way of simulating fluids under shear. Not only can one examine the viscometric properties of the fluid, both close and far from equilibrium, but one can also study the effect of shear on local fluid microstructure, on molecular conformation and upon molecular motion including rotation and internal vibration.

If the number of particles in the periodic cell is very small so that the range of the inter-atomic potential is comparable to the unit cell dimensions, it has been noticed that the time-periodicity implicit in the Lees-Edwards periodic boundary conditions influences statistical averages of phase functions. The long time state becomes time-periodic [3], exhibiting oscillations with a period inversely proportional to the shear rate, $\gamma$. The amplitude of oscillations decreases very rapidly when the number of particles in the unit cell, $N$, is increased. In our preliminary investigations of the number dependence of the amplitude of oscillations, we find that the amplitude of the shear stress oscillations is proportional to $N^{-\lambda}$ (Fig.1), where the exponent $\lambda$ does not depend on shear rate, but depends on density, interaction potential and Cartesian dimension of the system. Power laws with similar exponents are found for the number dependence of the amplitude of oscillations in the pressure and in the normal stress difference.

For larger systems, for example 72 soft discs per unit cell and 256 spherical WCA [4] particles per unit cell [5], such oscillations were not observed because their amplitude is negligible. In this case, the linear response could be correctly obtained from the Green Kubo expressions. Indeed the earliest methods for calculating the transport coeffiicents of fluids employed the Green-Kubo relations. The natural, thermostatted, nonlinear generalisation of Green-Kubo theory, valid for autonomous systems, is the Transient Time-Correlation Function formalism (TTCF) [1]. The nonlinear response of the SLLOD system calculated using direct simulation and the TTCF method in [5] showed excellent statistical agreement between the two methods for the two moderately large systems. This numerical test established the usefulness and correctness of the TTCF response theory, 
which was shown to yield the correct statistical averages of phase functions with an efficiency which is, at least for weak fields, superior to direct simulation.

For systems in which the unit cell is small compared to the range of the interaction potential, both the Green Kubo linear response theory and the nonlinear response theory for autonomous systems (TTCF and equivalent Kawasaki [6] formalism), fail to reproduce the oscillations in the shear stress and pressure obtained in the direct simulation results. We have previously investigated the oscillatory time dependence of the phase functions for the smallest possible system of two particles [7], where the oscillations are of the largest possible amplitude. In the present paper we shall show that the amplitude of these oscillations is a non-linear function of the applied shear rate. In spite of this nonlinearity, we show that at non-zero frequency the standard Green-Kubo relation for the linear, frequency dependent shear viscosity is incorrect for small systems undergoing 'steady' shear flow.

Recently we developed a generalisation of nonlinear response theory (TTCF) which can be applied to systems subject to time-periodic external fields [8,9]. Although the time-dependence of the final $(t \rightarrow \infty)$ state in the SLLOD system is not due to a time dependent shear, but rather is the inevitable consequence of Lees-Edwards periodic boundary conditions, we show that the time-dependent TTCF formalism can be modified so that it can be applied to shear flow. An unexpected bonus is that even for the strongest shear rates studied in this paper, this extended TTCF formalism enables the calculation of the response with greater computational efficiency than is possible by direct averaging of computer simulation results. 


\section{TIME DEPENDENCE IN LEES-EDWARDS PERIODIC BOUNDARY CONDITIONS}

The SLLOD equations of motion for a two dimensional system of $N$ particles are

$$
\begin{aligned}
\dot{\mathbf{q}}_{i} & =\mathbf{p}_{i} / m+\mathbf{i} \gamma y_{i}, \\
\dot{\mathbf{p}}_{i} & =\mathbf{F}_{i}-\mathbf{i} \gamma p_{y i}-\alpha \mathbf{p}_{i},
\end{aligned}
$$

where $\mathbf{p}_{i}$ is the peculiar momentum of the particle $i$ and $\mathbf{q}_{i}$ its position in a laboratory frame (see Fig.2). The parameter $\gamma$ is the shear rate $\left(\gamma=\partial u_{x} / \partial y\right.$, where $\mathbf{u}=\mathbf{i} u_{x}$ is the streaming velocity) and $\alpha$ is the time dependent Gaussian thermostat, multiplier

$$
\alpha=\frac{\sum_{i=1}^{N}\left(\mathbf{F}_{i} \cdot \mathbf{p}_{i}-\gamma p_{x i} p_{y i}\right)}{\sum_{i=1}^{N} p_{i}^{2}},
$$

which ensures the conservation of the peculiar kinetic energy, $\sum p_{\mathrm{i}}^{2} / 2 m$, at all times. This form of thermostat assumes that a linear streaming velocity profile, $\mathbf{u}=\gamma y$, is stable. This assumption is valid at low Reynolds number.

$\mathbf{F}_{i}$ is the sum of pair interactions between particle $i$ and all the other particles within its minimum image cell,

$$
\mathbf{F}_{i}=\sum_{i \neq j} \mathbf{F}_{i j}
$$

We shall use the WCA interaction potential defined as the Lennard-Jones potential truncated at the position of minimum potential energy $r_{i j}=2^{1 / 6} \sigma$, where $r_{i j}$ is the distance between pairs, and then shifted so that the potential is zero at the cutoff,

$$
U_{i j}^{\mathrm{WCA}}= \begin{cases}4 \varepsilon\left[\left(\sigma / r_{i j}\right)^{12}-\left(\sigma / r_{i j}\right)^{6}\right]+\varepsilon & \text { for } r_{i j}<2^{1 / 6} \sigma \\ 0 & \text { for } r_{i j} \geq 2^{1 / 6} \sigma .\end{cases}
$$

We work with a dimensionless system where the particle mass $m$ and the Lennard-Jones potential parameters $(\sigma$ and $\varepsilon$ ) are all set to unity. 
The Lees-Edwards periodic boundary conditions [10] define the motion of the neighbouring periodic cells to be consistent with the linear streaming velocity profile in the equations of motion (1). This does not imply that the $x$-component of the streaming velocity is linear in the $y$-coordinate; only that at zero wavevector the strain rate is consistent with the strain rate appearing in the equations of motion (1). Of course if the Reynolds number is small the observed velocity profile will be linear.

The motion of the unit cell images is such that their individual origins move with an $x$-velocity proportional to the $y$ coordinate of the particular cell origin. If $L$ is the sidelength of the square periodic cell, the relative displacement $d_{x} L$ of the origin of its neighbour on top (Fig.2) will depend on time as

$$
d_{x}(t)=\bmod \left(d_{x}(0)+\gamma t, 1\right) .
$$

This causes the configuration of the periodic cells to change periodically in time with the period of $1 / \gamma$. We shall sometimes refer to this configuration as a 'lattice' or an 'array' of periodic cells. Note that $d_{x}(t)$ is the Lees-Edwards periodic lattice strain and $\gamma$ is the corresponding lattice strain rate. For continuous dynamics, the lattice strain rate must be equal to the strain rate appearing in the SLLOD equations of motion (1).

Since the interaction of particles is determined by the minimum image convention (Fig.2), the time dependent periodic boundary conditions (2) affect the interaction between particles periodically in time. In other words, in order to determine the sum of pair interactions $\mathbf{F}_{i}$ between the particle $i$ and all the other particles within its minimum image cell, we must know not only the coordinates of all particles within the primitive periodic cell, but also the Lees-Edwards lattice strain, $d_{x}(t)$, which defines the relative displacement of the nearest neighbour periodic cells, $d_{x}(t) L$. Therefore in the SLLOD equations (1) it is necessary to write $\mathbf{F}_{i}(t)$ instead of $\mathbf{F}_{i}$ because, in general, the particle interactions depend on the instantaneous configuration of the Lees-Edwards lattice. In this sense the SLLOD equations for shearing a Lees-Edwards lattice are nonautonomous.

One might think that the Lees-Edwards periodic boundary conditions are somehow intervening in the system dynamics. However, there is another way of viewing shearing 
periodic systems which shows that there is no such 'intervention'. Consider an equilibrium system which is periodic with respect to a fixed cubic lattice - the usual Bornvon Karman periodic boundary conditions. There are two ways of viewing this system. One could consider the dynamics of the $\mathrm{N}$ particles that populate the primitive cell and then employ periodic boundary conditions as a 'book keeping' device to conserve particle number when particles attempt to exit from the primitive cell. Alternatively one could consider (at least in principle) the motion of an infinite system of particles, that happens (at some initial time) to be spatially periodic, with $\mathrm{N}$ particles per unit cell. If one studies the time evolution of this infinite periodic system without reference to boundary conditions, the motion of any unit cell of $\mathrm{N}$ particles will be identical to the time evolution computed for a cell of $\mathrm{N}$ particles alone, but subject to periodic boundary conditions. In other words the dynamics is incapable of breaking the initial lattice symmetry of the infinite periodic array of particles.

For Lees-Edwards boundary conditions under SLLOD dynamics the same is true: the motion of a primitive cell of $\mathrm{N}$ particles under SLLOD dynamics (with or without thermostatting) employing Lees-Edwards periodic boundary conditions, is identical to the motion one would observe for an infinite, initially periodic array of particles evolving under SLLOD but without reference to the boundary conditions. If the initial infinite system is periodic with a superimposed linear velocity profile at some initial time, SLLOD dynamics will preserve the periodicity forever [1]. This is the reason why we say that Lees-Edwards periodic boundary conditions are the natural generalisation of periodic boundary conditions to shear flow.

Since the particle interactions depend on the instantaneous configuration of the Lees-Edwards lattice, the pair distribution function $\mathrm{g}\left(\mathbf{r}, d_{x}\right)$ of a sheared system becomes different for different instantaneous configurations, as shown in Fig.3a for the twodimensional 2 particle case at the density of $\rho=0.396850$, for $d_{x}=0$ (square configuration) and for $d_{x}=L / 2$ (triangular configuration) when the shear rate is $\gamma=2$. In equilibrium, for $\gamma=0$, when the configuration of periodic cells is stationary, we cannot observe any variation in $\mathrm{g}\left(\mathbf{r}, d_{x}\right)$ with respect to $d_{x}$ from the plot obtained by simulation 
(Fig.3b). We expect that this is a special property of two particle systems, for which there is no interaction between a particle and any of its images at the reduced density $\rho=$ 0.396850 used in our simulations.

In shear flow simulations the quantities of greatest interest are the ensemble averages of the microscopic expressions for the elements of the pressure tensor. In the two dimensional case the shear stress $P_{x y}$ is

$$
\begin{aligned}
P_{x y} & =\frac{1}{V}\left\langle\sum_{i=1}^{N}\left(\frac{p_{x i} p_{y i}}{m}-\frac{1}{2} \sum_{\substack{j=1 \\
j \neq i}}^{N} x_{i j} F_{y i j}\left(x_{i}, x_{j}, y_{i}, y_{j}, d_{x}\right)\right)\right\rangle \\
& =\frac{1}{V}\left\langle\sum_{i=1}^{N}\left(\frac{p_{x i} p_{y i}}{m}-\frac{1}{2} \sum_{\substack{j=1 \\
j \neq i}}^{N} x_{i j} F_{y i j}\left(x_{i j}, y_{i j}\right)\right)\right\rangle
\end{aligned}
$$

and the hydrostatic pressure is given by

$$
P=\frac{1}{2}\left(P_{x x}+P_{y y}\right)=\frac{1}{2 V}\left\langle\sum_{i=1,2}\left(\frac{p_{x i}^{2}+p_{y i}^{2}}{m}-\frac{1}{2} \sum_{j=1,2} x_{i j} F_{x i j}\left(x_{i j}, y_{i j}\right)\right)\right\rangle .
$$

In these expressions $x_{i}$ and $y_{i}$ are the $x$ and $y$ coordinates of particle $i$ within the primitive cell, $x_{i j}$ and $y_{i j}$ are the $x$ and $y$ components of the relative distance between the discs in the minimum image cell of particle $i$, and $F_{x i j}$ and $F_{y i j}$ are the $x$ and $y$ components of the force on $i$ due to $j$. In general one cannot compute the minimum image separation of two particles (nor the force between two particles), without also knowing the lattice strain. Because $g(\mathbf{r})$ and the pair interactions are influenced by the periodic boundary conditions, it can be expected that even in the long time limit $t \rightarrow \infty$, the steady state for these quantities will not exist, and their ensemble averages will be time-periodic.

The shear stress $-P_{x y}$ in Fig. 4 was obtained by direct computer simulation of 2 WCA disks at the density of $\rho=0.396850$ and for two shear rates $\gamma=1$ and $\gamma=2$. Its periodic time dependence is obvious, and it appears in both the potential part which depends on position, and in the kinetic part which depends only on peculiar momentum and is therefore not an explicit function of the lattice strain. The period of oscillation is equal to $1 / \gamma$, and therefore when the shear rate is doubled, the frequency is also doubled, 
as can be observed in Fig.4. The same periodicity can be observed for the hydrostatic pressure.

In Fig.5a we see that the amplitude is, for sufficiently small strain rates, a quadratic function of the applied strain rate. Fig.5a shows the dependence of the amplitude of oscillations of the shear stress and the pressure in the time-periodic state on the strain rate. The values of the shear stress or the pressure, averaged over one period of oscillation at long times (Fig.5b) can be related to the steady state values of these functions in the systems subject to constant shear. The period averaged shear stress is a linear function of $\gamma$ at low fields while the pressure is a nonlinear function of $\gamma$, since the shear induced increase in pressure, shear dilatancy, is an intrinsically nonlinear effect. Green-Kubo linear response theory predicts a linear response for shear stress, while the Green-Kubo predicted change in pressure is exactly zero.

We shall show below that the evolution of the average of some function $B\left(\mathbf{q}_{i}, \mathbf{p}_{i}, d_{x}\right), i=1, \ldots, N$, from its equilibrium value to the final periodic state can be described using the generalised time dependent transient time-correlation function formalism for time-periodic external fields described in $[8,9]$. This formalism has to be modified to allow for the fact that the time dependence in this system arises not from an external field (constant shear) but rather from the boundary conditions and the implied interatomic forces. 


\section{EXTENDED PHASE SPACE AVERAGES}

The state of a system is conventionally represented by a point in phase space $\Gamma$ spanned by $\left(\mathbf{q}_{i}, \mathbf{p}_{i} ; i=1, \ldots, N\right)$. If stationary periodic boundary conditions are used (for example, Born-von Karman), the microscopic expressions of interest are phase functions, i.e. functions of phase space coordinates only. However, when Lees-Edwards time dependent boundary conditions are used, all functions of relative distances between particles cease to be phase functions and become explicit functions of $d_{x}(t)$. therefore it is convenient to define the extended phase space $\Gamma^{\prime}=\left\{\Gamma, d_{x}\right\}$ with an additional coordinate $d_{x}$. The state of the system is then completely represented by a point in extended phase space, and the components of the pressure tensor and other quantities of interest are extended phase functions.

The extended phase space has a meaning even in equilibrium, when $\gamma=0$, because we can consider stationary configurations of periodic cells characterised by different constant values of $d_{x}$. Equilibrium trajectories which start on a particular extended phase space hyperplane with $d_{x}=D$ are confined to this hyperplane forever. Since the equilibrium extended phase space probability distribution $f_{0}^{\prime}\left(\Gamma^{\prime}\right)$ depends on the potential energy of pair interaction, and therefore on $d_{x}$, it is different on different hyperplanes. The values of an extended phase function $B\left(\Gamma^{\prime}\right)=B\left(\boldsymbol{\Gamma}, d_{x}\right)$ at extended phase space points with the same coordinate $\Gamma$ and different coordinates $d_{x}$ can also be different.

The equilibrium extended phase space average of $B\left(\Gamma^{\prime}\right)$ is defined as

$$
\langle B(t=0)\rangle^{\prime}=\int d \boldsymbol{\Gamma}^{\prime} B\left(\boldsymbol{\Gamma}^{\prime}\right) f_{0}^{\prime}\left(\boldsymbol{\Gamma}^{\prime}\right)=\int d \boldsymbol{\Gamma}^{\prime} d d_{x} B\left(\boldsymbol{\Gamma}, d_{x}\right) f_{0}^{\prime}\left(\boldsymbol{\Gamma}, d_{x}\right),
$$

and the phase space average for a specified value of $d_{x}=D$,

$$
\langle B(D ; t=0)\rangle=\int d \boldsymbol{\Gamma}^{\prime} B\left(\boldsymbol{\Gamma}^{\prime}\right) f_{0}^{\prime}\left(\boldsymbol{\Gamma}^{\prime}\right) \delta\left(d_{x}-D\right)=\int d \boldsymbol{\Gamma} B\left(\boldsymbol{\Gamma}, d_{x}=D\right) f_{0}^{\prime}\left(\boldsymbol{\Gamma}, d_{x}=D\right) .
$$


The equilibrium averages do not depend on time, they are denoted by $t=0$ because for $t \leq 0$ the system is assumed to be at equilibrium, while for $t>0$ shear is applied and the system is not at equilibrium.

When shear is introduced into the equations of motion, the boundary conditions are no longer stationary and the trajectories are no longer confined to hyperplanes of constant $d_{x}$. They move along the $d_{x}$ axis at a constant rate of $\dot{d}_{x}=\gamma$. The probability distribution changes from the equilibrium distribution $f_{0}^{\prime}\left(\boldsymbol{\Gamma}^{\prime}\right)$ to the nonequilibrium extended phase space distribution $f^{\prime}\left(\Gamma^{\prime}, t\right)$, which in the long time limit becomes time independent. This nonequilibrium steady state exists only in the extended phase space. The equations of motion (1), with $\mathbf{F}_{i}$ explicitly dependent on time, would give a timedependent (in this case time-periodic) nonequilibrium distribution function in the conventional phase space $\Gamma$ even in the long-time limit.

The extended phase space average of $B\left(\Gamma^{\prime}\right)$ outside equilibrium is

$$
\langle B(t)\rangle^{\prime}=\int d \Gamma^{\prime} B\left(\Gamma^{\prime}\right) f^{\prime}\left(\Gamma^{\prime}, t\right)=\int d \Gamma d d_{x} B\left(\Gamma, d_{x}\right) f^{\prime}\left(\Gamma, d_{x}, t\right),
$$

and the phase space average on a particular hyperplane $d_{x}=D$ outside equilibrium is

$$
\begin{aligned}
\langle B(D ; t)\rangle & =\int d \boldsymbol{\Gamma}^{\prime} B\left(\boldsymbol{\Gamma}^{\prime}\right) f^{\prime}\left(\boldsymbol{\Gamma}^{\prime}, t\right) \delta\left(d_{x}(t)-D\right) \\
& =\int d \boldsymbol{\Gamma} B\left(\boldsymbol{\Gamma}, d_{x}(t)=D\right) f^{\prime}\left(\boldsymbol{\Gamma}, d_{x}=D, t\right) .
\end{aligned}
$$

Both of the above averages depend on time during the transient period of $f^{\prime}$, and become time independent in the long time limit.

The approach to the nonequilibrium steady state from different starting configurations of periodic cells is illustrated in Fig.6. Here we show the shear stress $\left\langle P_{x y}\left(d_{x}(t) ; t\right)\right\rangle$ responses of two 2-disk systems, one starting at $d_{x}=0$ at $t=0$ (rectangular configuration, full line) and the other at $d_{x}=0.5$ at $t=0$ (triangular configuration, dotted line), subjected to the shear rate $\gamma=1$. At short times (during the transient period) the dependence of $\left\langle P_{x y}\right\rangle$ on $d_{x}$ and $t$ is different for the two configurations, because they reach the same values of $d_{x}$ at different times. However, the long-time responses differ 
only in a phase lag of half a period. In the long time limit the value of the response (in this case $\left.P_{x y}\right)$ does not depend on the initial $d_{x}$ at $t=0$, but is a unique function of $d_{x}(t)$.

The equations of motion (1), which contain explicit time dependence, become autonomous in the extended phase space,

$$
\begin{aligned}
& \dot{\mathbf{q}}_{i}=\mathbf{p}_{i} / m+\mathbf{i} \gamma y_{i}, \\
& \dot{\mathbf{p}}_{i}=\mathbf{F}_{i}\left(d_{x}\right)-\mathbf{i} \gamma p_{y i}-\alpha\left(d_{x}\right) \mathbf{p}_{i}, \\
& \dot{d}_{x}=\gamma .
\end{aligned}
$$

The shear starts to act at $t=0$ upon the equilibrium system in the configuration of periodic cells characterised by $d_{x}(0)$.

While the oscillations in $\langle B\rangle$ present in small systems under constant shear are an artefact of the boundary conditions, the evolution of the extended phase space average of $B\left(\Gamma^{\prime}\right)$ can be related to the values of this phase function in real systems. The extended phase space average at long times is the same as the average over one period in Fig.5. The average over the extended phase space of a phase function $\mathrm{B}$, taken at time $t>0$, is

$$
\begin{aligned}
\left\langle B\left[\boldsymbol{\Gamma}^{\prime}(t)\right]\right\rangle^{\prime} & =\int d \boldsymbol{\Gamma}^{\prime} f^{\prime}\left(\boldsymbol{\Gamma}^{\prime}, t\right) B\left(\boldsymbol{\Gamma}^{\prime}\right) \\
& =\int d \boldsymbol{\Gamma}^{\prime} f^{\prime}\left(\boldsymbol{\Gamma}^{\prime}, 0\right) B\left[\boldsymbol{\Gamma}^{\prime}(t)\right] \\
& =\int d \boldsymbol{\Gamma} d d_{x} f_{0}^{\prime}\left(\boldsymbol{\Gamma}^{\prime}\right) B\left[\boldsymbol{\Gamma}^{\prime}(t)\right],
\end{aligned}
$$

in the Schrödinger and Heisenberg pictures, respectively. We shall use the Heisenberg picture.

The equation of motion for $B\left(\Gamma^{\prime}\right)$ can be obtained using the chain rule,

$$
\frac{d B\left[\boldsymbol{\Gamma}^{\prime}(t)\right]}{d t}=\dot{\boldsymbol{\Gamma}} \cdot \frac{\partial}{\partial \boldsymbol{\Gamma}}\left[B\left(\boldsymbol{\Gamma}^{\prime}\right)\right]+\gamma \frac{\partial}{\partial d_{x}}\left[B\left(\boldsymbol{\Gamma}^{\prime}\right)\right] .
$$

Differentiation of the Heisenberg expression (6) for $\langle B(t)\rangle^{\prime}$ using (7) yields

$$
\frac{d\left\langle B\left[\boldsymbol{\Gamma}^{\prime}(t)\right]\right\rangle^{\prime}}{d t}=\int d \boldsymbol{\Gamma}^{\prime} f_{0}^{\prime}\left(\boldsymbol{\Gamma}^{\prime}\right)\left(\dot{\boldsymbol{\Gamma}}^{\prime} \cdot \frac{\partial B\left(\boldsymbol{\Gamma}^{\prime}\right)}{\partial \boldsymbol{\Gamma}^{\prime}}\right) .
$$

In order to obtain (8) the order of differentiation with respect to time and integration over extended phase space has to be interchanged. This is allowed if the 
operator $\dot{\Gamma}^{\prime} \cdot\left(\partial / \partial \Gamma^{\prime}\right)$ does not depend explicitly on time. Since the equations of motion for $\Gamma^{\prime}$ in the extended phase space (5) contain no explicit time dependence, this condition is satisfied. Integrating (8) by parts we get

$$
\frac{d\left\langle B\left[\Gamma^{\prime}(t)\right]\right\rangle^{\prime}}{d t}=-\int d \Gamma^{\prime} B\left[\Gamma^{\prime}(t)\right]\left[\frac{\partial}{\partial \Gamma^{\prime}} \cdot\left(\dot{\Gamma}^{\prime} f_{0}^{\prime}\left(\Gamma^{\prime}\right)\right)\right],
$$

since the boundary term vanishes.

The time-independent equilibrium extended phase space probability distribution of the isokinetic system is

$$
f_{0}^{\prime}\left(\boldsymbol{\Gamma}^{\prime}\right)=\frac{\exp \left[-\beta U\left(\boldsymbol{\Gamma}, d_{x}\right)\right] \delta\left(K(\boldsymbol{\Gamma})-K_{0}\right)}{\int d \boldsymbol{\Gamma}^{\prime} \exp \left[-\beta U\left(\boldsymbol{\Gamma}, d_{x}\right)\right] \delta\left(K(\boldsymbol{\Gamma})-K_{0}\right)},
$$

where $U$ is the potential energy of the system, which is the sum of the pair potentials $U_{i j}$,

$$
U\left(\Gamma, d_{x}\right)=\frac{1}{2} \sum_{\substack{i, j=1 \\ i \neq j}}^{N} U_{i j}\left(\mathbf{r}_{i}, \mathbf{r}_{j}, d_{x}\right),
$$

$K_{0}=d N / 2 \beta$ is the kinetic energy, $\beta=1 / k_{B} T$ where $T$ is the temperature, $k_{B}$ the Boltzmann constant and $d$ is the Cartesian dimensionality of the system. Using expression (10) we can evaluate the term in the rectangular brackets in (9),

$$
\frac{\partial}{\partial \boldsymbol{\Gamma}^{\prime}} \cdot\left[\dot{\boldsymbol{\Gamma}}^{\prime} f_{0}^{\prime}\left(\boldsymbol{\Gamma}^{\prime}\right)\right]=\left.\frac{\partial}{\partial \boldsymbol{\Gamma}} \cdot\left[\dot{\boldsymbol{\Gamma}} f_{0}^{\prime}\left(\boldsymbol{\Gamma}^{\prime}\right)\right]\right|_{d_{x}=\text { const. }}+\left.\gamma \frac{\partial f_{0}^{\prime}\left(\boldsymbol{\Gamma}^{\prime}\right)}{\partial d_{x}}\right|_{\Gamma=\text { const. }} .
$$

The second term on the right hand side is

$$
\left.\gamma \frac{\partial f_{0}^{\prime}\left(\boldsymbol{\Gamma}^{\prime}\right)}{\partial d_{x}}\right|_{\Gamma=\text { const. }}=-\beta \gamma f_{0}^{\prime}\left(\boldsymbol{\Gamma}^{\prime}\right) \frac{\partial U}{\partial d_{x}}=-\beta \gamma f_{0}^{\prime}\left(\boldsymbol{\Gamma}^{\prime}\right) \frac{\partial}{\partial d_{x}} \frac{1}{2} \sum_{\substack{i, j=1 \\ i \neq j}}^{N} U_{i j}\left(\mathbf{r}_{i}-\mathbf{r}_{j}, d_{x}\right)
$$

The physical meaning of this term is the rate of change of $f_{0}^{\prime}\left(\boldsymbol{\Gamma}^{\prime}\right)$ with the change in $d_{x}$ if all the positions and momenta of particles within the periodic cell are kept constant. The distribution function can change only if the pair potential energy $U_{i j}$ changes with the change in $d_{x}$. Since the positions of particle images depend on $d_{x}$, the potential energy $U_{i j}$ can depend on $d_{x}$ if the relative distance $r_{i j}$ of particles $i$ and $j$ within the unit cell is greater than $L / 2$. Let us suppose that particle $i$, located at $y_{i}$, interacts with the image of particle $j$, namely with particle $j^{\prime}$, located at $x_{j^{\prime}}=x_{j}+L d_{x}, y_{j^{\prime}}=y_{j}+L$. Then somewhere inside 
the unit cell particle $j$, at $x_{j}, y_{j}$ interacts with the image of $i$, i.e. particle $i^{\prime \prime}$ at $x_{i^{\prime \prime}}=x_{i}-L d_{x}, y_{i^{\prime \prime}}=y_{i}-L$. Therefore we obtain,

$$
\begin{aligned}
\frac{\partial}{\partial d_{x}} \frac{1}{2}\left[U_{i, j}\left(\mathbf{r}_{i}-\mathbf{r}_{j^{\prime}}, d_{x}\right)+U_{j, i}\left(\mathbf{r}_{j}-\mathbf{r}_{i^{\prime \prime}}, d_{x}\right)\right] \\
=\frac{\partial U_{i, j}\left(\mathbf{r}_{i}-\mathbf{r}_{j^{\prime}}, d_{x}\right)}{\partial x_{j^{\prime}}} \frac{\partial x_{j^{\prime}}}{\partial d_{x}}+\frac{\partial U_{j, i}\left(\mathbf{r}_{j}-\mathbf{r}_{i^{\prime \prime}}, d_{x}\right)}{\partial x_{i^{\prime \prime}}} \frac{\partial x_{i^{\prime \prime}}}{\partial d_{x}} \\
=\frac{1}{2}\left[-F_{i j x} L-F_{j i x}(-L)\right]=-\frac{1}{2} F_{i j x} L .
\end{aligned}
$$

The second term of the right hand side of Eq. (11) is therefore the sum of the $x-$ components of the forces on the particles within a periodic cell which interact with images of particles across an $y$-boundary of the periodic cell.

The left hand side of (11) is, from the Loiuville equation in the extended phase space [1],

$$
\frac{\partial}{\partial \boldsymbol{\Gamma}^{\prime}} \cdot\left[\dot{\boldsymbol{\Gamma}}^{\prime} f_{0}^{\prime}\left(\boldsymbol{\Gamma}^{\prime}\right)\right]=-\frac{\partial}{\partial t} f_{0}^{\prime}\left(\boldsymbol{\Gamma}^{\prime}\right)=\beta f_{0}^{\prime}\left(\boldsymbol{\Gamma}^{\prime}\right) \frac{\partial}{\partial t} U\left(\boldsymbol{\Gamma}, d_{x}\right),
$$

which is for isokinetic SLLOD system equal to

$$
\beta f_{0}^{\prime}\left(\boldsymbol{\Gamma}^{\prime}\right) \frac{\partial}{\partial t} U\left(\boldsymbol{\Gamma}, d_{x}\right)=\beta \gamma V P_{x y}\left(\boldsymbol{\Gamma}, d_{x}\right) f_{0}^{\prime}\left(\boldsymbol{\Gamma}, d_{x}\right) .
$$

The first term on the right hand side is the contribution to shear stress when $d_{x}$ is fixed, and the second term the contribution when $\mathbf{q}, \mathbf{p}$ are fixed. The total shear stress $-P_{x y}$, is the sum of these two terms.

The equation of motion (9) finally reduces to

$$
\begin{aligned}
\frac{d\left\langle B\left[\boldsymbol{\Gamma}^{\prime}(t)\right]\right\rangle^{\prime}}{d t} & =-\beta \gamma V \int d \boldsymbol{\Gamma}^{\prime} f_{0}^{\prime}\left(\boldsymbol{\Gamma}^{\prime}\right) B\left[\boldsymbol{\Gamma}^{\prime}(t)\right] P_{x y}\left[\boldsymbol{\Gamma}^{\prime}(0)\right] \\
& =-\beta \gamma V\left\langle B\left[\boldsymbol{\Gamma}^{\prime}(t)\right] P_{x y}\left[\boldsymbol{\Gamma}^{\prime}(0)\right]\right\rangle^{\prime},
\end{aligned}
$$

with the solution

$$
\left\langle B\left[\Gamma^{\prime}(t)\right]\right\rangle^{\prime}=\left\langle B\left[\Gamma^{\prime}(0)\right]\right\rangle^{\prime}-\beta \gamma V \int_{0}^{t} d s\left\langle B\left[\Gamma^{\prime}(t)\right] P_{x y}\left[\Gamma^{\prime}(0)\right]\right\rangle^{\prime} .
$$

In particular, if we choose $B\left[\Gamma^{\prime}(t)\right] \equiv P_{x y}\left[\Gamma^{\prime}(t)\right]$, the expression for the time evolution of the extended phase space average of shear stress $P_{x y}$ is: 


$$
\left\langle P_{x y}\left[\boldsymbol{\Gamma}^{\prime}(t)\right]\right\rangle^{\prime}=\left\langle P_{x y}\left[\Gamma^{\prime}(0)\right]\right\rangle^{\prime}-\beta \gamma V \int_{0}^{t} d s\left\langle P_{x y}\left[\Gamma^{\prime}(t)\right] P_{x y}\left[\Gamma^{\prime}(0)\right]\right\rangle^{\prime}
$$

The simulation results for a 2 disk system at the strain rate of $\gamma=1$ are shown in Fig.7. The simulations were done at the density of $\rho=0.396850$ and at the temperature of $T=1.0$, using the fourth-order Runge-Kutta method of integration of the equations of motion (5) with a timestep of $\delta t=0.005$. The interval [0,1] of possible values of $d_{x}$ has been divided into 50 subintervals of width $\delta d_{x}=0.02$, and the timestep of time integration in (13) is therefore $d s=0.02$. From each starting phase $\Gamma^{\prime}=\left(\mathbf{q}_{i}, \mathbf{p}_{i} ; d_{x}\right)$ of the isokinetic equilibrium ensemble, three additional starting points were generated using the timereversal mapping $\mathbf{M}^{\mathrm{T}}$, the y-reflection mapping $\mathbf{M}^{\mathrm{Y}}$, and the Kawasaki mapping $\mathbf{M}^{\mathrm{K}}$ $[1]$

$$
\begin{gathered}
\mathbf{M}^{\mathrm{T}}\left(\boldsymbol{\Gamma}^{\prime}\right)=\left(\mathbf{q}_{i},-\mathbf{p}_{i} ;-d_{x}\right), \\
\mathbf{M}^{\mathrm{Y}}\left(\boldsymbol{\Gamma}^{\prime}\right)=\left(x,-y, z, p_{x},-p_{y}, p_{z} ; d_{x}\right), \\
\mathbf{M}^{\mathrm{K}}\left(\boldsymbol{\Gamma}^{\prime}\right)=\left(x,-y, z,-p_{x}, p_{y},-p_{z} ;-d_{x}\right),
\end{gathered}
$$

in order to improve the statistics. These additional starting phase points ensure that the average initial shear stress is identically zero. Since the objective of this simulation has been to test whether the direct calculations and our theory (13) coincide, we used a large number of initial trajectories, $4 \times 20000$ for each of the 50 values of $d_{x}(0)$. The results of the two simulations coincide in Fig.7. 


\section{OSCILlations OF THE PHASE SPACE AVERAgES}

The generalised TTCF theory can also reproduce oscillations of the averages of phase functions in small systems undergoing steady shear, such as shown in Fig.4.

As can be seen from Fig.6, the long time behaviour of a phase function average $\langle B(t)\rangle$ can be regarded as $\langle B(t)\rangle$ having a different "steady state" for each $d_{x}(t) \in[0,1]$. The approach to this state for any particular value $d_{x}=D$ can be derived from the expression for the phase space average of $B$ with $d_{x}=D$ at time $t$, analogous to the Heisenberg picture in (6),

$$
\begin{aligned}
\left\langle B\left[\Gamma(t), d_{x}(t)=D\right]\right\rangle & =\left\langle B\left[\Gamma^{\prime}(t)\right] \delta\left[d_{x}(t)-D\right]\right\rangle^{\prime} \\
& =\int d \Gamma^{\prime} f_{0}^{\prime}\left(\Gamma^{\prime}\right) B\left[\Gamma^{\prime}(t)\right] \delta\left(d_{x}(t)-D\right) .
\end{aligned}
$$

Integration of (14) over the whole extended phase space would give

$$
\left\langle B\left[\Gamma(t), d_{x}(t)=D\right]\right\rangle=\int d \boldsymbol{\Gamma} f_{0}^{\prime}\left[\boldsymbol{\Gamma}(0), d_{x}(0)=D-\gamma t\right] B\left[\Gamma(t), d_{x}(t)=D\right],
$$

because in Heisenberg picture the values of $B$ are taken at time $t$ at the coordinates $\boldsymbol{\Gamma}(t)$ on the hyperplane $d_{x}(t)=D$, and the probability density $f_{0}^{\prime}$ is taken at the coordinates $\Gamma(0)$ on a hyper plane $d_{x}(0)=D-\gamma t$ from which the points $\Gamma^{\prime}(t)$ originated.

Differentiating (14) with respect to time, we find using the same procedure as in Section 3,

$$
\begin{aligned}
\frac{d}{d t}\left\langle B\left[\boldsymbol{\Gamma}^{\prime}(t)\right] \delta\left(d_{x}(t)-D\right)\right\rangle^{\prime}=\int d \boldsymbol{\Gamma}^{\prime} f_{0}^{\prime}\left(\boldsymbol{\Gamma}^{\prime}\right) \dot{\boldsymbol{\Gamma}}^{\prime} \cdot \frac{\partial}{\partial \boldsymbol{\Gamma}^{\prime}}\left[B\left(\boldsymbol{\Gamma}^{\prime}(t)\right) \delta\left(d_{x}(t)-D\right)\right] \\
\quad=-\int d \boldsymbol{\Gamma}^{\prime} B\left[\boldsymbol{\Gamma}^{\prime}(t)\right] \delta\left[d_{x}(t)-D\right] \frac{\partial}{\partial \boldsymbol{\Gamma}^{\prime}} \cdot\left[\dot{\boldsymbol{\Gamma}}^{\prime} f_{0}^{\prime}\left(\boldsymbol{\Gamma}^{\prime}\right)\right] \\
\quad=-\beta \gamma V \int d \boldsymbol{\Gamma}^{\prime} B\left[\boldsymbol{\Gamma}^{\prime}(t)\right] \delta\left[d_{x}(t)-D\right] P_{x y}\left[\boldsymbol{\Gamma}^{\prime}(0)\right] f_{0}^{\prime}\left[\boldsymbol{\Gamma}^{\prime}(0)\right] \\
\quad=-\beta \gamma V \int d \boldsymbol{\Gamma} B\left[\boldsymbol{\Gamma}(t), d_{x}(t)=D\right] P_{x y}\left[\boldsymbol{\Gamma}(0), d_{x}(0)=D-\gamma t\right] f_{0}^{\prime}\left[\boldsymbol{\Gamma}(0), d_{x}(0)=D-\gamma t\right] \\
\quad=-\beta \gamma V\left\langle B\left(\boldsymbol{\Gamma}(t) ; d_{x}(t)=D\right) P_{x y}\left(\boldsymbol{\Gamma}(0), d_{x}(0)=D-\gamma t\right)\right\rangle .
\end{aligned}
$$

Integrating this equation gives,

$$
\begin{aligned}
\left\langle B\left[\Gamma(t), d_{x}(t)=D\right]\right\rangle & =\left\langle B\left[\Gamma(0), d_{x}(0)=D\right]\right\rangle \\
& -\beta \gamma \int_{0}^{t} d s\left\langle B\left[\Gamma(s), d_{x}(s)=D\right] P_{x y}\left[\Gamma(0), d_{x}(0)=D-\gamma s\right]\right\rangle .
\end{aligned}
$$


The right hand side of the equation (15), $\left\langle B\left(\Gamma(t) ; d_{x}(t)=D\right)\right\rangle$, means the average over all values of the phase $\Gamma$, at the time $t$, for a particular chosen configuration of periodic cells characterised by $d_{x}=D$ at time $t$, i.e. $d_{x}(t)=D$. The first term on the left hand side of (15) is the equilibrium average of $B$ calculated for the stationary configuration characterised by $d_{x}=D$. In the second term, the expression under the time integral is a correlation function of $B$ at time $s$, such that $d_{x}(s)$ is equal to $D$, and $P_{x y}$ evaluated from the same trajectory at $t=0$, when $d_{x} \operatorname{was} \bmod (D-\gamma s, 1)$. This means that for all times $s \in[0, t], d_{x}(s)$ has to be a constant equal to $D$, and that we need to use trajectories starting at different initial arrays of periodic cells to obtain the same type of configuration at the time $s$. In other words, in order to find the evolution of $\left\langle B\left[\boldsymbol{\Gamma}(t), d_{x}(t)=D\right]\right\rangle$ for the chosen value of $d_{x}(t)=D$, we need to know the behaviour of trajectories starting from the configurations with all possible initial symmetries at all previous times.

The response of the shear stress $-P_{x y}$ has been monitored as a function of the shift of neighbour cells $d_{x}$ and time $t$. It has been calculated from the expression corresponding to $(15)$,

$$
\begin{aligned}
\left\langle P_{x y}\left[\Gamma(t), d_{x}(t)=D\right]\right\rangle & =\left\langle P_{x y}\left[\Gamma(0), d_{x}(0)=D\right]\right\rangle \\
& -\beta \gamma V \int_{0}^{t} d s\left\langle P_{x y}\left[\Gamma(s), d_{x}(s)=D\right] P_{x y}\left[\Gamma(0), d_{x}(0)=D-\gamma s\right]\right\rangle .
\end{aligned}
$$

The conditions and parameters of simulation were as described at the end of Section 3.

When the shear stress correlation function in (16) is integrated over the same time $t$ for many different configurations $d_{x}=D$, the dependence of $\left\langle P_{x y}\right\rangle$ on $d_{x}$ at time $t$ is obtained. This dependence first changes its form, and after a long time (in this case the time of $5 / \gamma$ was found to be sufficient), when the system reaches its steady state in the extended phase space, the form of the $d_{x}$ dependence remains constant in time. The evolution of the shear stress response as a function of $d_{x}$, evaluated by direct simulation and using the generalised TTCF formula (16), is shown in Fig.8. After the system has been subjected to shear for a short time ( $t=0.5$ in Fig.8) the variations of $\left\langle P_{x y}\right\rangle$ with the 
change of $d_{x}$ are small. Later the amplitude of oscillations increases until a final state pattern is established.

Fig.9 shows the approach to the steady state of $\left\langle P_{x y}\left[\Gamma(t), d_{x}(t)=D\right]\right\rangle$ for four constant values of values of $d_{x}=D$. The generalised TTCF results were obtained using formula (15).

In Figs 10.a and 10.b, the phase space average of $P_{x y}$ has been evaluated from the trajectories starting from the same equilibrium configuration, with $d_{x}(0)=0$ in the former and $d_{x}(0)=0.5$ in the latter, and was followed over five periods of the lattice symmetry change. These graphs show the real responses to shear starting from a given equilibrium configuration. There is no closed generalised TTCF expression for this type of response, but formula (15) has to be applied at each timestep with the initial equilibrium configuration such that at time $s$, the lattice is characterised by $d_{x}=d_{x}(0)+\gamma s$

In all these results, there is a good correspondence between direct and TTCF calculations, but there is less noise present in the TTCF results. Noise reduction is a feature typical of all TTCF methods [9]. 


\section{THE LINEAR LIMIT}

The expression (15) is a generalised expression for the time-dependent response in the time periodic boundary conditions in the SLLOD model of steady shear flow. The linear time dependent response formula [1], applicable in the low shear rate limit, is obtained from (15) if the equilibrium correlation function is substituted for the transient correlation in the integrand of (15), keeping in mind that in equilibrium, the periodic cells are stationary and $d_{x}$ does not depend on time. Therefore in the linear limit we obtain

$$
\begin{aligned}
\left\langle B\left[\Gamma(t), d_{x}(t)=D\right]\right\rangle & =\left\langle B\left[\Gamma(0), d_{x}(0)=D\right]\right\rangle_{\mathrm{eq}} \\
& -\beta \gamma V \int_{0}^{t} d s\left\langle B\left[\Gamma(s), d_{x}(s)=D\right] P_{x y}\left[\Gamma(0), d_{x}(0)=D\right]\right\rangle_{\mathrm{eq}}
\end{aligned}
$$

where the subscript ' $e q$ ' denotes an equilibrium ensemble average.

Unlike the nonlinear formula (15), where the contributions to the integrand at different times $s$ come from families of trajectories starting from different initial $d_{x}(0)$, in (17) the equilibrium correlation function in the integrand is evaluated from the same set of trajectories in a stationary configuration characterised by $d_{x}=D$ at all times $s$. Equation (17) shows how to calculate the linear response of a phase function at time t, when the Lees-Edwards lattice strain, $d_{x}(\mathrm{t})$, is $D$ (at each time of observation, $t$ ) from a nonshearing equilibrium system with a fixed lattice strain $d_{x}=D$ at all times $s$. The oscillatory response in the long time limit $(t \rightarrow \infty)$ can be obtained from (17) if we allow $d_{x}$ on the left hand side to vary in time as $d_{x}(t)=d_{x}(0)+\gamma t$, and at each time $t$ we evaluate the correlation integral on the right hand side in the equilibrium configuration with $D=d_{x}(t)$, i.e. the instantaneous value of $d_{x}(t)$ on the left hand side. The long time oscillations would be present in the linear limit if the second term on the right hand side depended on $D$ for $t \rightarrow \infty$. The equation (17) is local in the lattice strain with the lattice strain for the fixed equilibrium correlation function, equal to the instantaneous value of the nonequilibrium lattice strain. This is easily understood since in the limit of low shear rate $\gamma \rightarrow 0$, the period of oscillation $1 / \gamma$ becomes infinitely long compared to the time required for convergence of the Green-Kubo time integral. 
The results of equilibrium simulations of the shear stress $-P_{x y}$, in the two particle system described above, for two configurations with $d_{x}=0$ and $d_{x}=0.5$, are shown in Fig.11. In both cases, the equilibrium correlation function in (17) has been calculated from $2 \times 10^{6}$ trajectories using the shift register technique [11]. In our calculations the shift register was not filled at every timestep, but rather we employed a waiting period of 1000 timesteps between the starting points of old and new trajectories (ie filling the shift register).

One can immediately see that the transient response for the two experiments is different. This does not mean that linear response is ambiguous. Rather it simply reflects the fact that the two different responses are for two different experiments. In the first one the shear stress at time $\mathrm{t}$ is predicted for systems where the lattice strain at is zero at all times $t$, and in the other experiment the lattice strain at the time $B(\mathrm{t})$ is observed, is always 0.5. These are different experiments and one should not be surprised that the response curves are different.

In Figure 11 we see that although the transient linear response is dependent on the lattice strain, at long times the integral of the equilibrium time correlation function appearing in (17), appears to be independent of the lattice strain at which the correlation function is calculated. From (17) this implies that in the zero strain rate, long time limit, the ensemble averaged shear stress, $-\left\langle P_{x y}\left[d_{x}(t)=D\right]\right\rangle$, is independent of the lattice strain. This is consistent with the fact that the amplitude of the stress oscillations (see Fig.5a), is a nonlinear function of the shear rate. Because the dependence of the response on $d_{x}$ and on $\gamma$ cannot be separated, the integral of the equilibrium stress autocorrelation function (17) is independent of the Lees-Edwards strain in the long time limit, and the steady state linear response of the stress is unaffected by the nonautonomous terms implicit in the Lees-Edwards dynamics. 


\section{CONCLUSION}

We have pointed out that for 'steady' shear flow under Lees-Edwards shearing periodic boundary conditions, the equations of motion are in fact non-autonomous, and that the microscopic expressions for the quantities of interest, such as elements of the pressure tensor, are not simply functions of the coordinates and momenta of all the primitive cell particles for this model. One in fact needs to make explicit reference to time before a unique ensemble averaged value can be defined for this model.

We have shown numerically that for short ranged potentials, the effects of these non-autonomous terms decrease extremely rapidly with increasing system size, $N$. We expect however, that for systems with long ranged potentials this will not be so. The possible implications of this observation for Coulombic systems is an open question.

We have also shown numerically that in the long time limit, the effect of these non-autonomous terms is nonlinear and that they therefore have no effect on the long time (zero frequency), linear response of the system - even if the system is small relative to the range of the interatomic potential. However, the standard Green-Kubo expression for the linear response of Lees-Edwards systems which makes no reference to the lattice strain, and therefore is incorrect for predictions of the transient response. We have derived a corrected Green-Kubo expression for the linear response of the Lees-Edwards system, which takes proper account of the time varying, Lees-Edwards lattice strain.

Likewise in the nonlinear regime conventional (autonomous) response theory for steady shear flow is inapplicable and we have developed a generalisation of time dependent nonlinear response theory which successfully describes Lees-Edwards shear flow for a constant strain rate.

We have tested this theory against nonequilibrium computer simulation and found excellent agreement between theory and experiment. This agreement is all the more impressive because of the irregular and complex shapes of the response curves. The 
chance of fortuitous agreement between theory and experiment must therefore be very small.

An added bonus is that when our theory is used in conjunction with computer simulations to estimate the response, one obtains estimates which are more accurate than those obtained by directly averaging the observed response. It is important to note in this efficiency comparison both the direct averaging and the response theory calculations employ exactly the same molecular dynamics simulations. The only difference lies in how the data from those simulations is processed. This improved efficiency is apparent for all shear rates studied in this paper, even the highest shear rate.

\section{ACKNOWLEDGEMENTS}

We wish to thank Professor W. G. Hoover for a useful discussion of the Green-Kubo theory. Evans would like to thank the staff of the NIST Physical and Chemical Properties Division for their hospitality. 


\section{REFERENCES:}

[1] D. J. Evans and G. P. Morriss, Statistical Mechanics of Nonequilibrium Liquids, (Academic Press, New York, 1990).

[2] S.Y. Liem, D. Brown and J. H. R. Clarke, Phys. Rev. A 45, 3706 (1992).

[3] W. G. Hoover and B. Moran, Phys. Rev A 40, 5319 (1989)

[4] J. D. Weeks, D. Chandler and H. C. Andersen, J. Chem. Phys. 54, 5237 (1971).

[5] D. J. Evans and G. P. Morriss, Phys. Rev. A 38, 4142 (1988)

[6] D. J. Evans and D. J. Searles, Phys. Rev. E 52, 5839 (1995)

[7] J. Petravic, D. J. Isbister and G. P. Morriss, J. Stat. Phys 76, 1405 (1994)

[8] J. Petravic and D. J. Evans, Phys. Rev. Letts. 78, 1199 (1997)

[9] J. Petravic and D. J. Evans, Phys. Rev. E 56, 1207 (1997).

[10] A. W. Lees and S. F. Edwards, J. Phys. C5, 1921 (1975)

[11] M. P. Allen and D. J. Tildesley, Computer Simulation of Liquids, Clarendon Press, Oxford 1987. 
FIGURE CAPTIONS:

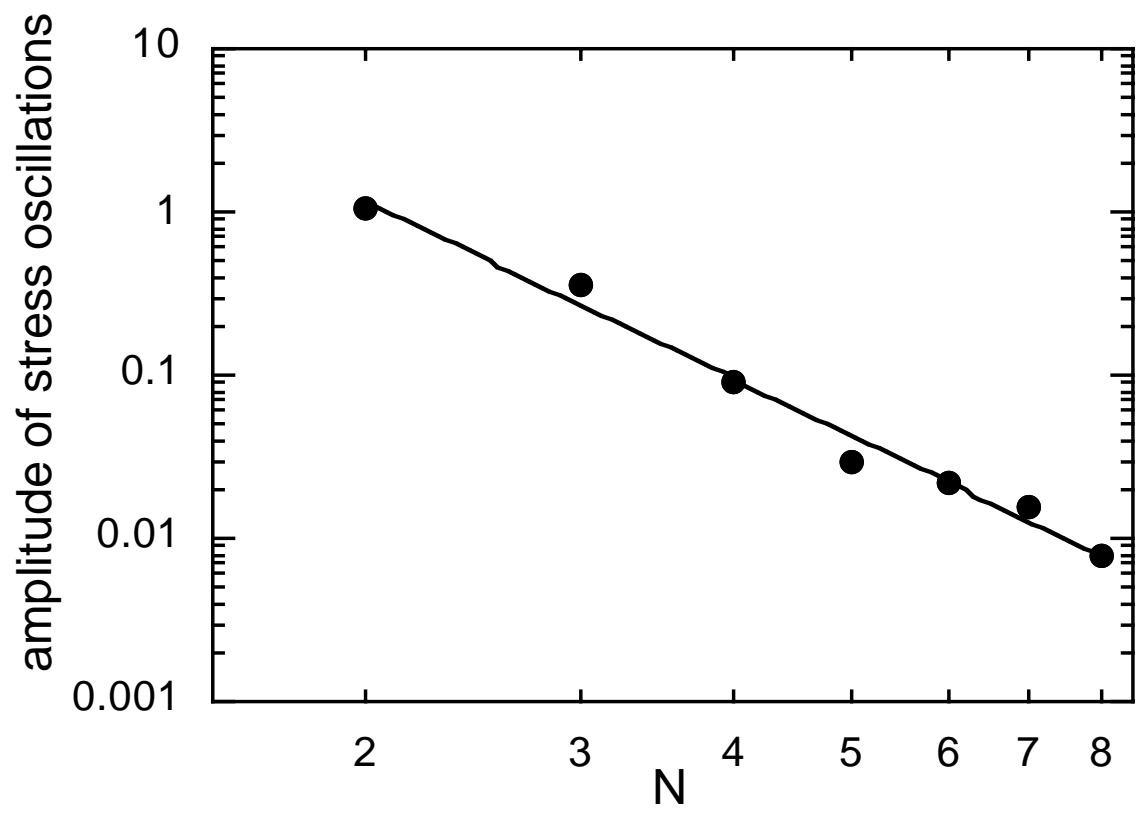

FIGURE 1. Number dependence of the amplitude of long-time oscillations of shear stress for a system of WCA discs of the density $\rho=0.396850$ at the temperature $T=1$, subjected to a shear rate $\gamma=2$. The curve fit shows that the amplitude decreases as $N^{-3.6}$. For $N=8$ the amplitude of the shear stress is already only $1.5 \%$ of its average value over one period, and therefore the oscillations are in fact negligible. The exponent of $N$ in the power law depends on density, interaction potential and cartesian dimension of the system. 


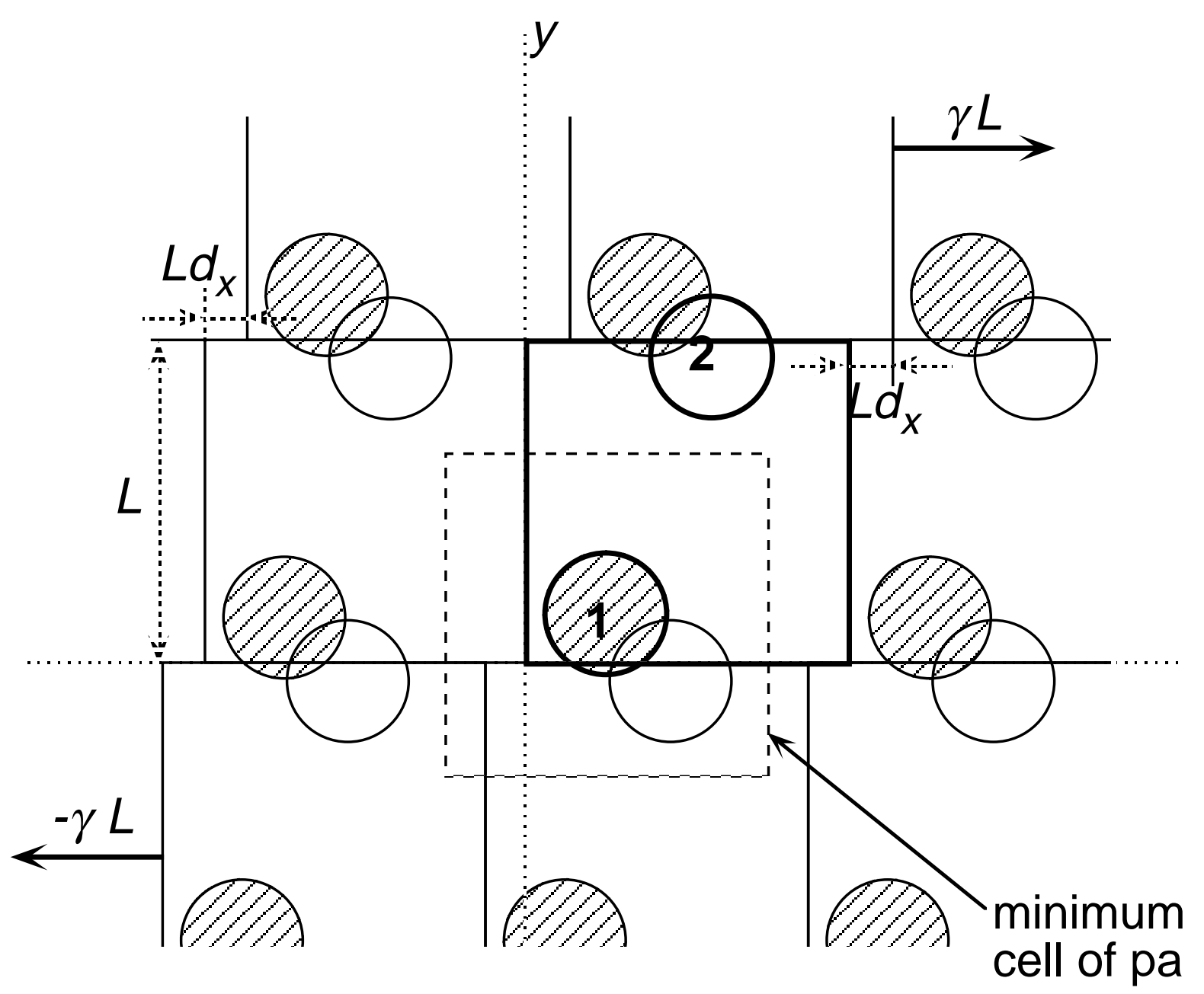

FIGURE 1. Petravic, Evans Phys. Rev 
FIGURE 2. Lees-Edwards periodic boundary conditions for planar shear flow. The $N$ interacting particles are placed in a cubic cell which is surrounded by an infinite array of identical cells. Each horizontal row of this array moves with horizontal velocity of $\gamma L$ with respect to the row immediately below it. Therefore the array of periodic cells constantly changes its symmetry. The instantaneous form of the array is characterised by the $x$ component of the displacement of the origin of the primitive cell and its nearest neighbour in $+y$ direction, $L d_{x}$. To compute a force on a given particle in the primitive cell, one has to locate the closest image positions of the other $N-1$ particles. Since the minimum image cell usually at least partially lies outside the primitive cell, one needs to know the displacement $L d_{x}$ to determine the positions of the image particles. 

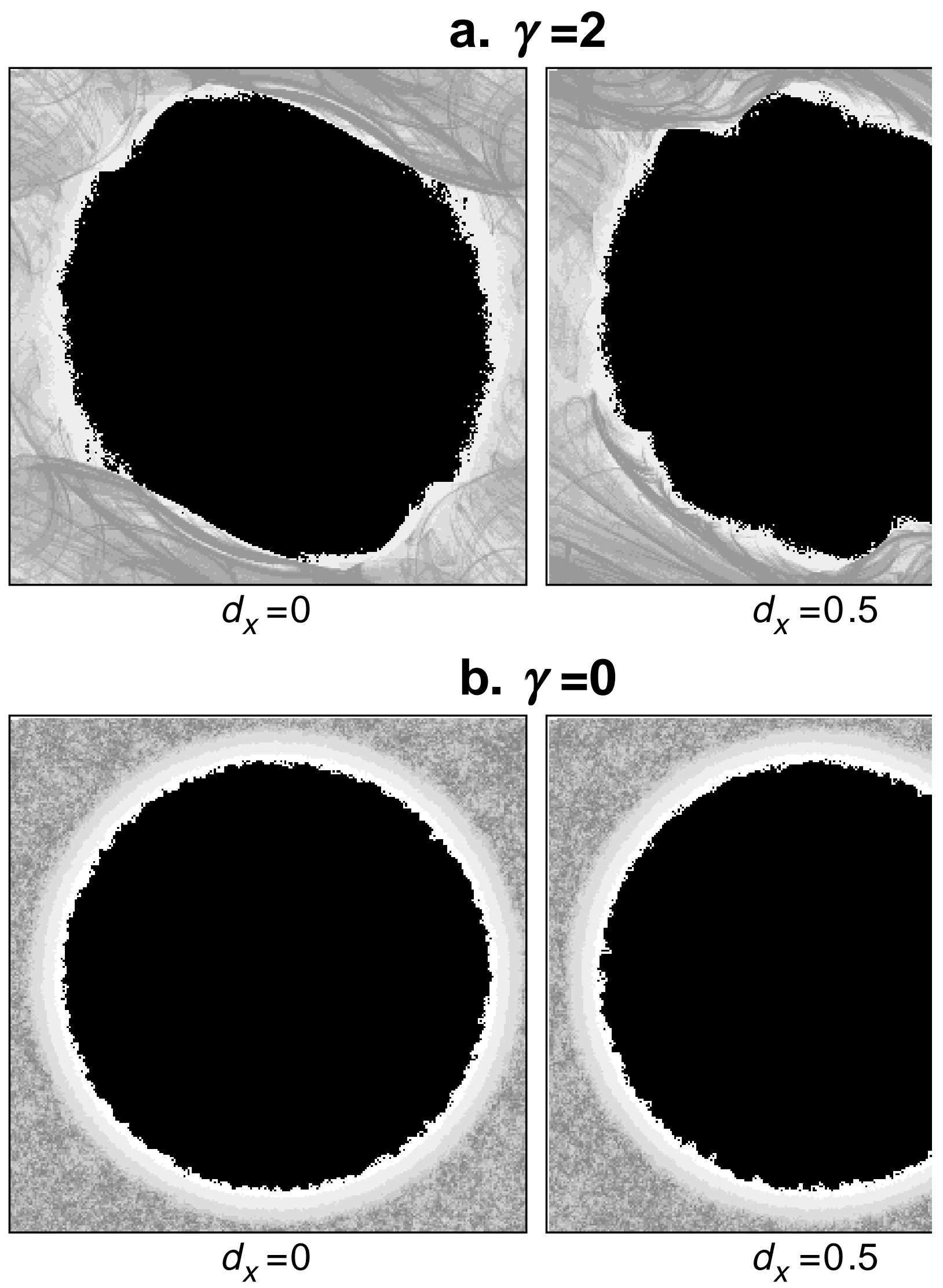

FIGURE 3. Petravic, Evans 
FIGURE 3. (a) When shear is present, the pair distribution functions $g(\mathbf{r})$ for the lattices of rectangular $\left(d_{x}=0\right)$ and triangular $\left(d_{x}=0.5\right)$ symmetry are different. (b) In equilibrium, $\mathrm{g}(\mathbf{r})$ does not seem to depend on symmetry of the lattice.
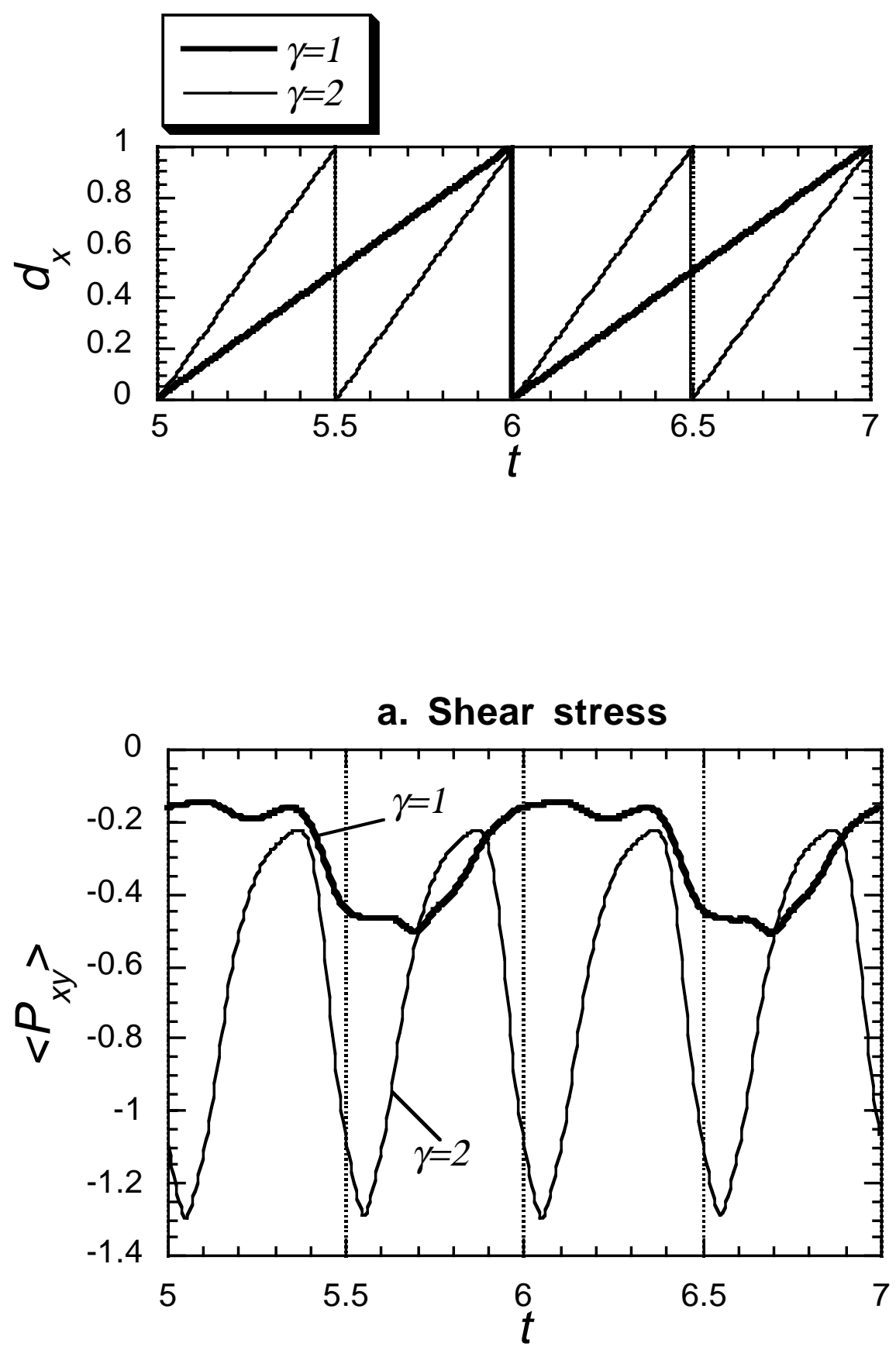


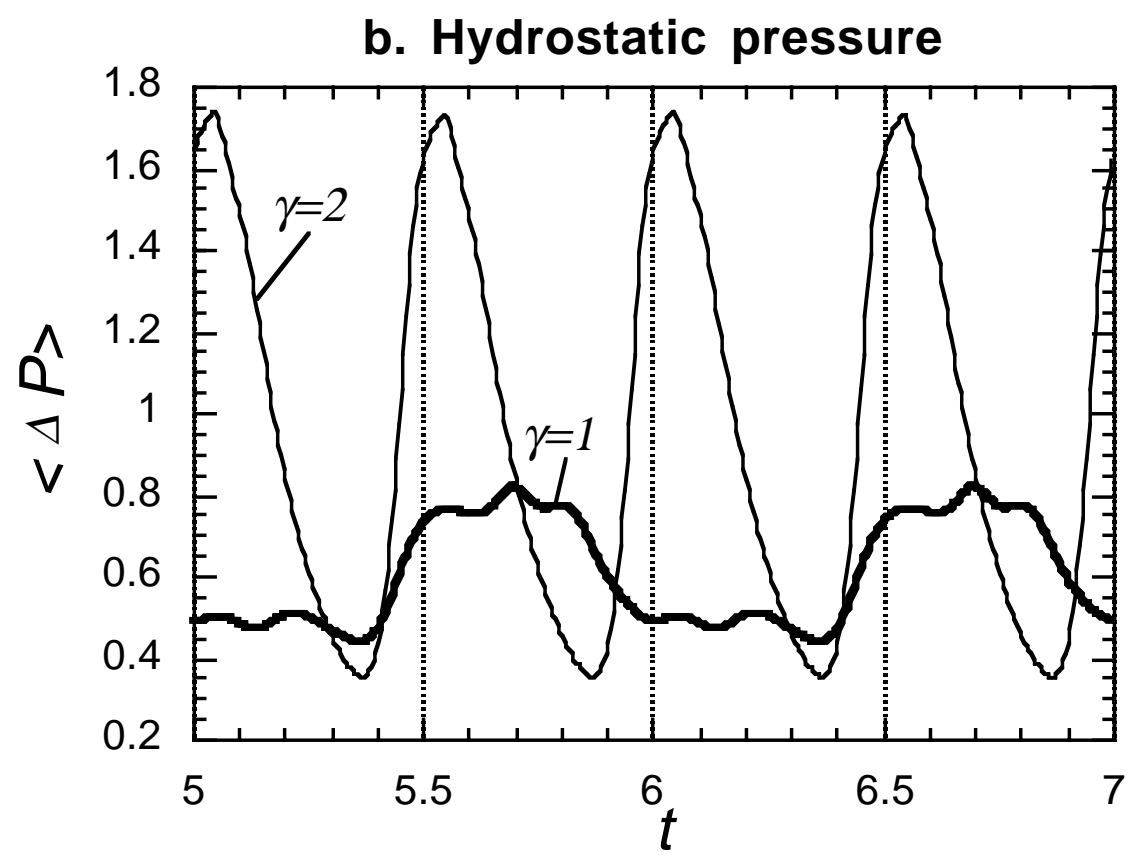

FIGURE 4. In the long time limit, the phase space average of shear stress of a 2particle system is periodic in time. The period is equal to $1 / \gamma$, the same as the period of the change of the configuration of periodic cells. 

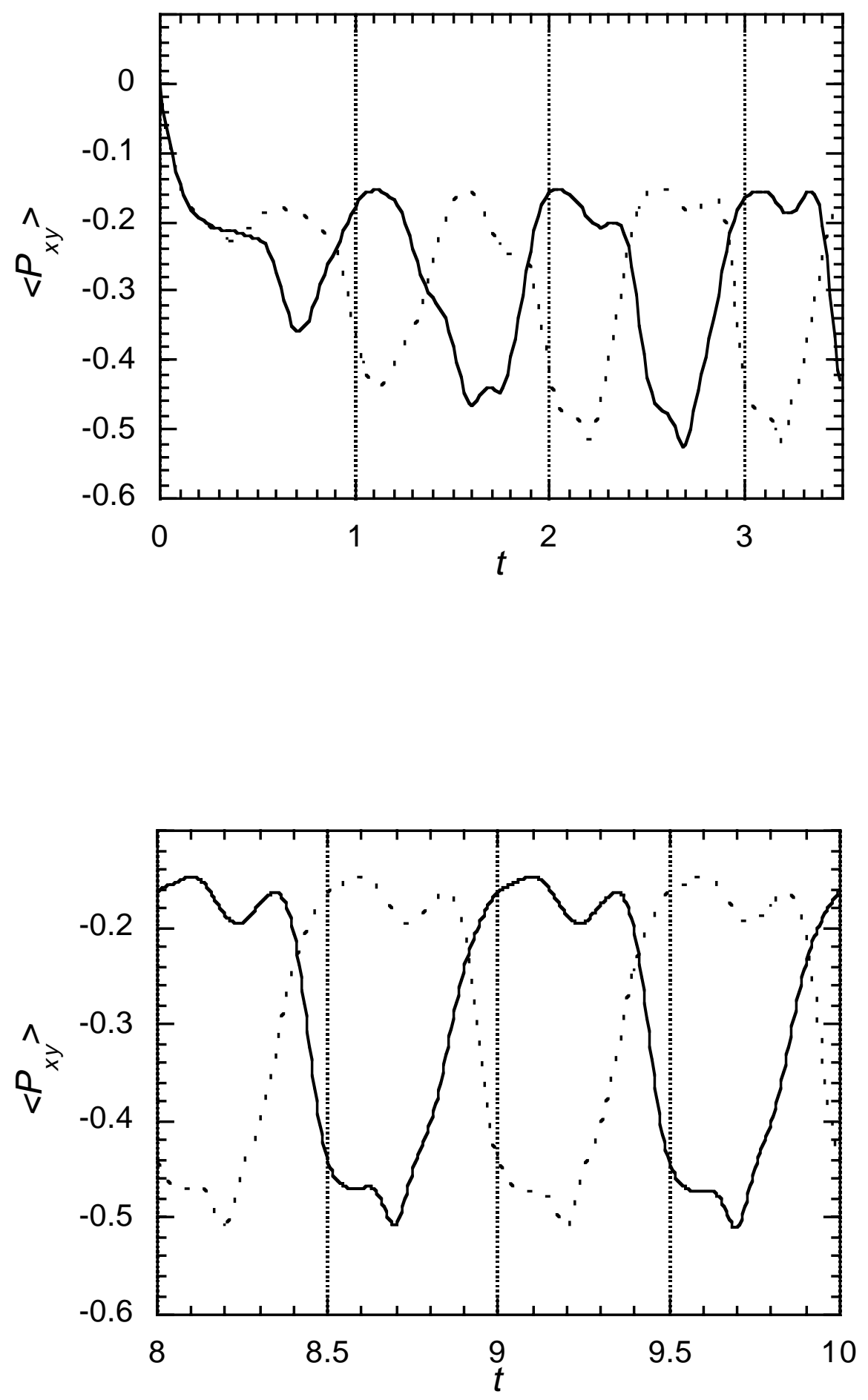

FIG

URE 5. (a) Dependence of the amplitude of oscillations of shear stress and hydrostatic pressure of a 2-disk system on the reduced shear rate is nonlinear even for low values of 
shear rate. (b) Dependence of these two phase functions, averaged over one period, on shear rate. Shear stress decreases linearly with the increase in $\gamma$ for low shear rates, but the change in hydrostatic pressure is entirely nonlinear.

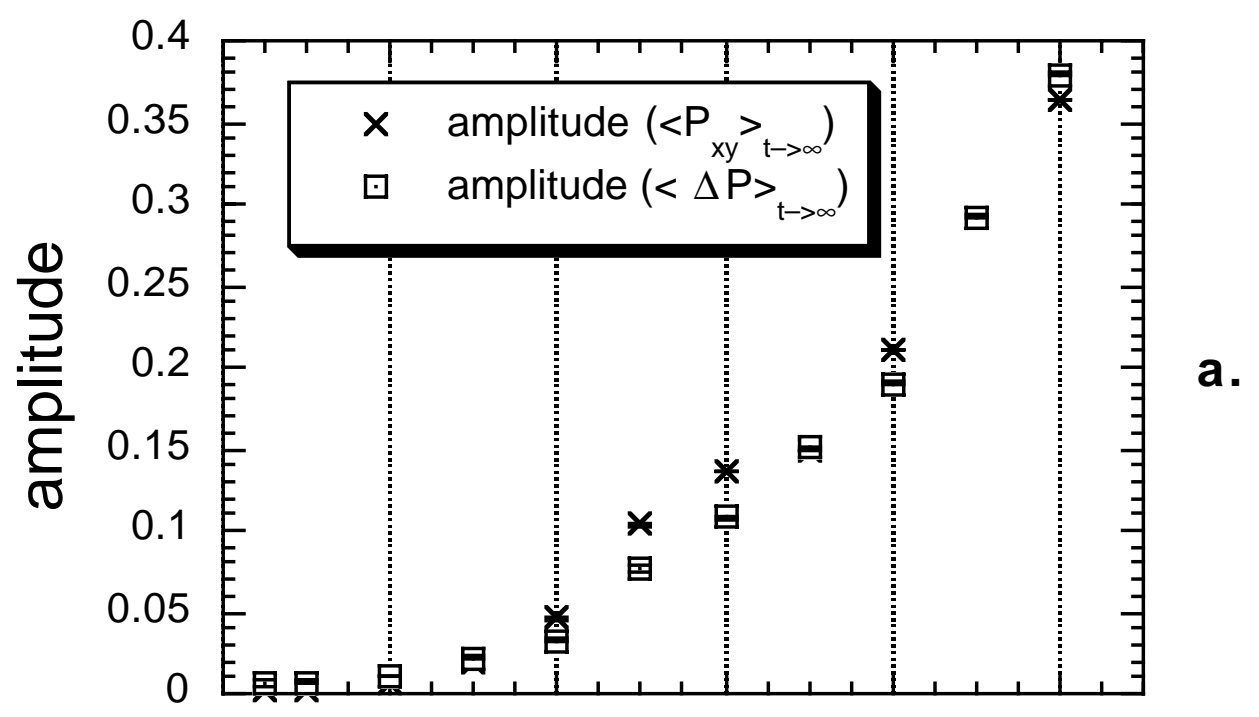

FIGURE 6. Evolution of the shear stress for the shear rate of $\gamma=1$, when the initial periodic array is rectangular $\left(d_{x}=0\right)$ and triangular $\left(d_{x}=0.5\right)$. (a) The initial transient behaviour is different. (b) In the long time limit, the time dependence is different, the patterns are shifted by $\Delta t=0.5$. (c) When $P_{x y}$ is considered a function of the instantaneous value of $d_{x}$, the long-time patterns coincide.

FIGURE 7. Time dependence of the extended phase space average of the shear stress of a two disc system with $\gamma=1$. The results of direct simulation and the generalised TTCF formalism coincide.

FIGURE 8 . The change of the $d_{x}$ dependence of the shear stress of a two disc system in time, evaluated using direct simulation and the generalised TTCF method.

FIGURE 9. The shear stress of a of a two disc system with $\gamma=1$ has different steady state values for different lattice configurations $d_{x}$. The approach to the steady state is calculated for four different configurations using direct simulation and the generalised 
TTCF method. The two methods show excellent correspondence of results, but there is less noise present in the TTCF results.

FIGURE 10. Direct simulation and TTCF results for the time evolution of the shear stress of a two disc system with $\gamma=1$, starting from two different lattice configurations, rectangular $\left(d_{x}(0)=0\right)$ and triangular $\left(d_{x}(0)=0.5\right)$. The two sets of results coincide in these graphs.

FIGURE 11. Steady state value of the shear stress of a two disc system with $\gamma=1$, for the rectangular $\left(d_{x}=0\right)$ and triangular $\left(d_{x}=0.5\right)$ lattice configurations, evaluated using the linear response theory. The results show that in the linear limit there are no oscillations in the shear stress. 